Brazilian Journal

of Chemical

Engineering

\title{
EXPERIMENTAL AND NUMERICAL ANALYSIS OF CHAOTIC ADVECTION AS AN EFFICIENT APPROACH TO MAXIMIZE HOMOGENEOUS LAMINAR MIXING IN A BATCH MIXER
}

\author{
F. Shirmohammadi ${ }^{1}$ and A. Tohidi ${ }^{1}$ \\ ${ }^{1}$ Islamic Azad University, Yadegar-e-Imam Khomeini (RAH) Shahre Rey Branch, Department of Mechanical Engineering, Tehran, Iran. \\ E-mail: tohidi@iausr.ac.ir - ORCID: 0000-0003-2072-448X
}

(Submitted: November 23, 2018 ; Revised: May 4, 2019 ; Accepted: May 28, 2019)

\begin{abstract}
In the present work, the impacts of effective parameters on the mixing of Stokes flows in a chaotic batch mixer are numerically and experimentally studied. The batch mixer consists of a container and two circular rotors where the rotors can rotate independently. To investigate the possibility of improving the mixing, the effect of nonconstant speeds, contra-rotating rotors, and varying rotor speeds are studied. The results showed that varying the rotor speed while rotating in the same direction does not significantly increase mixing. However, if the rotors rotate in opposite directions, 10-times more mixing is achieved compared to the mode of rotating in the same direction. Nevertheless, given the constant speed of rotors, the flow is steady since the fluid particles have periodic movements in secondary flows, but the flow becomes chaotic and mixing is considerably increased by applying sinusoidal perturbations to the rotor speed. Nevertheless, for both modes of rotations in the same and opposite directions, the chaotic flow leads to increased mixing index in the same amount of time. Based on results, the best mixing results are achieved when the rotors rotate with sinusoidal rotational speed in opposite directions.

Keywords: Stokes flow; Chaotic advection; Mixing; Laminar.
\end{abstract}

\section{INTRODUCTION}

The mixing process reduces heterogeneity in order to achieve the desired homogenous products. This heterogeneity can include concentration or temperature. The aim of the process is very important for successful production of a product. If the mixing is not appropriate, it can reduce the quality, physical properties and also increase the price. Although there are many industrial processes in mixing equipment, all these processes need to be evaluated as to whether the mixing amount is sufficient and the additional mixing results in yield and reduction of quality. It depends on the process and sensitivity of choice, physical properties, product stability and time. In the last few decades, effective methods for the mixing process in industrial laboratories have been obtained.
Homogenous laminar mixing is very difficult. In applications such as pharmaceuticals, food, polymers and biological processes, mixing of liquids is performed with low velocity or with high viscosity. In these cases, turbulence does not happen. Turbulence in highly viscous fluid is usually impossible and viscous energy dissipation results in unacceptable high temperatures in products. Laminar mixing needs to be used in highly viscous fluid such as pulp, soap, grease, and solid - made fluids.

One of the most important issues in flows with highly viscous fluids is the kinematic reversibility of these flows, i.e., if boundary conditions are inversed, the flow is inversed in all points. This property is only true for Stokes flows. The reversibility of Stokes flow leads to the non-homogenous mixing of two viscous fluids. In low viscosity fluids, turbulence will cause

\footnotetext{
* Corresponding author: A. Tohidi - E-mail: tohidi@iausr.ac.ir
} 
fluid to be properly mixed and consequently heat transfer will be improved. However, it is difficult to improve heat transfer in the cases where turbulent flow cannot be readily achieved. On the other hand, in the Stokes flows, it is necessary to find an appropriate solution for mixing, since they have a high dissipation rate of energy in a turbulent flow.

Chaotic advection has been considered as a useful solution to achieve a more effective mixing in high viscosity flows in the last two decades. Chaotic advection can be represented in two-dimensional timeperiodic flow and three - dimensional flows, which can be used as a useful tool to describe a simple flow under the influence of viscous forces. To analyze the mixing, the recognition of dynamical systems, particularly of chaos and its relation with flow kinematics is the most effective way. For this purpose, kinematic foundations of fluid mechanics can be combined with the concepts of dynamic systems, especially chaotic advection. In short, it can be stated that a system is chaotic that satisfies one of the following conditions: (Ottino and Ottino, 1989)

- The system is sensitive to initial conditions.

- The system creates a horseshoe mapping.

Two general approaches are used to describe problems in fluid mechanics: Lagrangian and Eulerian. The advection equations certainly belong to the Lagrangian perspective of fluid motion. From the standpoint of systems dynamics theory, any ordinary differential equation such as advection equations (1-2) is sufficient to generate the non-integrable and chaotic dynamics. There is no need to be dependent on time in 3D flow to make chaotic behavior and steady flow is sufficient, but to create chaotic motion of fluid particles in 2D flows, the flow must be time dependent, because the steady two dimensional advection equations of the particles are integrable (Aref, 2002).

$$
\begin{aligned}
& \frac{d x}{d t}=u(x, y) \\
& \frac{d y}{d t}=v(x, y)
\end{aligned}
$$

where $x$ and $y$ are positions. $t$ is time and $u$ and $v$ are velocity components.

The motion of a passive particle in a given velocity field can be considered from the viewpoint of dynamic systems theory (Zaslavsky, 1991). In chaotic advection, adjacent particles move away exponentially. This exponential divergence leads to stretching, which exponentially increases the contact surfaces of the fluid element, leading to a more efficient mixing. To obtain a three-dimensional chaotic flow, the flow does not have to be time-dependent and the steadiness of the flow is sufficient. However, to create the chaotic movement of particles in two-dimensional (2D) flows, the flow should be time-dependent. By creating a chaotic flow inside a highly viscous fluid mixer, the fluid is mixed homogenously.

So far, many attempts have been made to design a mixer with chaotic performance. The performance of the mixer is such that, by creating a perturbation in the flow, the flow path was changed and caused chaotic trajectories of fluid particles. Chaotic mixers can be categorized based on the type of perturbations caused by the flow into two main categories: (1) passive mixer (Aguirre et al., 2018, Grosso et al., 2018, Jung et al., 2018, Luan et al., 2018, Mizuno and Funakoshi, 2002, Mizuno and Funakoshi, 2004, Pacheco et al., 2006, Xu et al., 2016), which creates chaotic flow with a simple geometric perturbation and without input of energy, and (2) the active mixer (Jegatheeswaran et al., 2018, Tohidi et al., 2014, Tohidi et al., 2015, Tohidi et al., 2013, Wünsch and Böhme, 2000), which requires input energy to cause chaos in the flow.

Mixing enhancement by chaotic advection in an annulus flow between eccentric cylinders is an active mixer and of great interest for researchers. Jeffery (1922) and Ballal and Rivlin (1976) have obtained somewhat similar analytical solutions for this geometry. In such a 2D geometry, streamlines are more complicated compared to those of the flow between two concentric cylinders. In an experimental and numerical study, Swanson and Ottino (1990) showed that, in the flow between two eccentric cylinders, the temperature of the fluid inside the rotating area becomes uniform due to mixing in that area when one or both eccentric cylinders rotate with a constant speed. This factor can increase the Nusselt number by $50 \%$, indicating the ratio of convective heat transfer to conductive heat transfer.

The study conducted by Lefevre et al. (2003) consisted of two cylinders which the outer cylinder rotated with a constant speed while the rotation speed of the inner cylinder varied in a sinusoidal manner. The authors showed that mixing and heat transfer are improved due to chaotic advection when the cylinders rotate in a sinusoidal manner. Moreover, Niederkorn and Ottino (1994) numerically and experimentally investigated the mixing improvement in the annulus flow between two eccentric cylinders for nonNewtonian shear-thinning and viscoelastic fluids. They reported that the elasticity of the fluid in creeping flows increases or decreases the surface in which chaotic advection occurs. Furthermore, Galaktionov et al. (1999) analytically solved the 2D and steady Stokes flow inside a rectangular cavity with a cylinder inside it. They concluded that the resulting flow is chaotic due to the movement of the upper and lower walls of the rectangular cavity and the rotation of the cylinder and the mixing in this model is superior and more rapid compared to the cavity without a cylinder. 
Leprevost et al. (1997) introduced the area between two confocal ovals as a new geometry for mixing. They showed that, by selecting proper periodic speeds for the walls of the two ovals, the streamlines of the Stokes flow become chaotic and the particles can be diffused throughout the domain with a series of periodic oscillations. Due to a rapid mixing, the heat transfer rate is high in this model. In addition, El Omari and Le Guer (2010) studied the effect of chaotic mixing on heat transfer in a two-rotor mixer. They reported that discontinuous rotor speeds improve the mixing and heat transfer. They also showed that a better mixing is achieved with the rotation of the outer cylinder. Furthermore, Hosseinalipour et al. (2014, 2013) examined the mixing of a non-Newtonian flow inside a continuous chaotic mixer which consists of an eccentric helical rotor inside a cylindrical stator. They concluded that the eccentricity of the helical rotor and the stator leads to the formation of chaotic flow and, consequently, a better mixing. Msaad et al. (2017) studied the effect of the number of rotating rods on mixing and heat transfer. Their numerical results revealed that more rotating rods lead to more uniform mixing. They showed that non-continuous wall rotation is one of essential factors to generate a chaotic mixing.

The literature contains a number of devices designed to enhance mixing, but there is not a parametric study to maximize homogeneous laminar mixing in a batch mixer. This paper aims to fill this gap in the literature. The purpose of this paper was to numerically and experimentally examine the effect of chaotic flow on the mixing of a highly viscous fluid and reducing the time and energy necessary for achieving the required homogeneous mixing. In our previous study (Shirmohammadi and Tohidi, 2018), the effect of chaotic flow was studied on the mixing of highly viscous fluids. It was shown that mixing will be very poor if the rotors rotate with the same and constant speed. Therefore, the rotors are varied in the form of a sinusoidal function of time. With the perturbation generated in the rotational speed of the rotors, the secondary flow leading to the entrapment of fluid particles is eliminated and mixing is improved. In the present study, the effects of other effective parameters on the mixing of highly viscous fluids are numerically and experimentally examined. In this study, the effects of: (i) constant rotor speeds, (ii) unequal rotor speeds, (iii) rotations in the same and opposite direction, and (4) rotations of rotors with varying speeds are studied on mixing. The flow inside the mixer is simulated in two dimensions and in experiments, the mixing inside the mixer is studied as well and, by defining an index, the mixing inside the mixer is quantitatively compared for different modes.

\section{GEOMETRY OF THE STUDIED MIXER}

The geometry of the chaotic mixer is illustrated in Figure 1. The system consisted of a cylindrical reservoir with the internal diameter of $110 \mathrm{~mm}$ and two cylindrical rotors, each with the diameter of 30 $\mathrm{mm}$. The rotors were placed non-concentrically along the reservoir at the distance of $\mathrm{d}=27 \mathrm{~mm}$. Each of them was capable of independent rotation.

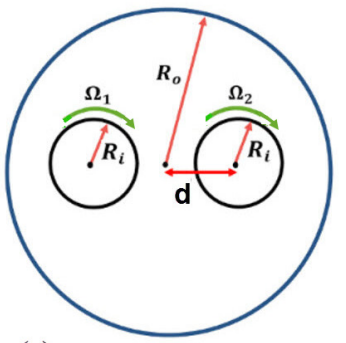

(a)

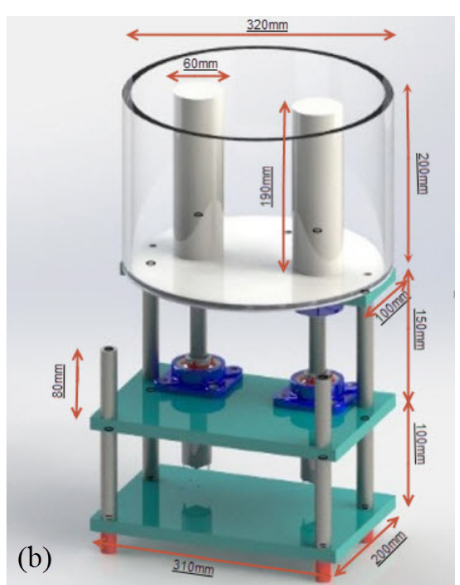

(b)
Figure 1. (a) Geometry of the batch mixer. (b) The design for the batch mixer created in Solidworks.

\section{BOUNDARY CONDITIONS}

Rotors can rotate with a rotational speed as expressed in Equations (3) and (4).

$\Omega_{1}=\mathrm{U}_{1}\left(1+\varepsilon_{1} \sin (\omega \mathrm{t})\right)$

$\Omega_{2}=\mathrm{U}_{2}\left(1+\varepsilon_{2} \sin (\omega \mathrm{t})\right)$

In these equations, $\omega=2 \pi / \mathrm{T}$ where $\mathrm{T}$ shows the value of temporal oscillations, which is considered to be $12 \mathrm{~s} ; \mathrm{U}_{1}$ and $\mathrm{U}_{2}$ are the mean rotational speeds of the rotors; and $\varepsilon_{1}$ and $\varepsilon_{2}$ are the perturbation applied to the rotor speeds, whose values are in the ranges of $0 \leq$ $\varepsilon_{1}$ and $\varepsilon_{2} \leq 1$. The performance of the mixer is studied in six modes:

- Mode 1: The rotational speeds of the rotors are assumed to be constant, equal, and in the same direction $\left(\mathrm{U}_{1}=20 \mathrm{rpm}, \mathrm{U}_{2}=20 \mathrm{rpm}, \varepsilon_{1}=0, \varepsilon_{2}=0\right)$.

- Mode 2: The rotational speeds of the rotors are assumed to be constant and in the same direction, but not equal $\left(\mathrm{U}_{1}=10 \mathrm{rpm}, \mathrm{U}_{2}=30 \mathrm{rpm}, \varepsilon_{1}=0, \varepsilon_{2}=0\right)$.

- Mode 3: The rotors rotate in the same direction, but their speed changes with time $\left(\mathrm{U}_{1}=20 \mathrm{rpm}, \mathrm{U}_{2}=\right.$ $20 \mathrm{rpm}, \varepsilon_{1}=0, \varepsilon_{2}=0.5$ ).

- Mode 4: The rotational speeds of the rotors are constant, equal, and in opposite directions $\left(\mathrm{U}_{1}=20\right.$ rpm, $\mathrm{U}_{2}=-20 \mathrm{rpm}, \varepsilon_{1}=0, \varepsilon_{2}=0$ ). 
- Mode 5: The rotational speeds of the rotors are constant, unequal, and in opposite directions $\left(\mathrm{U}_{1}=10\right.$ rpm, $\mathrm{U}_{2}=-30 \mathrm{rpm}, \varepsilon_{1}=0, \varepsilon_{2}=0$ ).

- Mode 6: The rotors rotate in opposite directions and their speed varies with time $\left(\mathrm{U}_{1}=20 \mathrm{rpm}, \mathrm{U}_{2}=\right.$ $20 \mathrm{rpm}, \varepsilon_{1}=0, \varepsilon_{2}=0.5$ ).

Equation 5 is defined to calculate the Reynolds number in the mixer geometry which contains the rotational speed of the cylinders (Jana et al., 1994):

$\mathrm{Re}=\sqrt{\left(\Omega_{1}^{2}+\Omega_{2}^{2}\right) \mathrm{R}_{\mathrm{i}}^{2}}\left(\mathrm{R}_{\mathrm{o}}-\mathrm{R}_{\mathrm{i}}\right) / \mathrm{v}$

where $\mathrm{R}_{\mathrm{i}}$ is the rotor radius, $\mathrm{R}_{\mathrm{o}}$ is the stator radius, $\Omega_{1}$ and $\Omega_{2}$ are the rotational speeds of the rotors, and $v$ is the kinematic viscosity of the fluid. The Strouhal number which contains the rotational speed of the cylinder, is defined as follows (Jana et al., 1994):

$\mathrm{St}=\left(\mathrm{R}_{\mathrm{o}}-\mathrm{R}_{\mathrm{i}}\right) / \sqrt{\left(\Omega_{\mathrm{A}}^{2}+\Omega_{\mathrm{B}}^{2}\right) \mathrm{R}_{\mathrm{i}}^{2}} \mathrm{~T}$

Given the applied boundary conditions, the Reynolds and Strouhal numbers vary from 13.2 to 16.7 and from 0.012 to 0.09 , respectively.

\section{EXPERIMENTAL TEST}

\section{Design and construction of the apparatus}

The mixer consists of two cylindrical rotors with different cross-sections and a stator. The motor specifications are SPG rs34-24v with a gearbox, 24volt $\mathrm{DC}$, and torque of $7.1 \mathrm{Nm}$. To constrain each axis, two UCF 204 bearings are used. A rotating cylinder made out of Teflon is employed. The distance of the center of the cylinder in this design is assumed to be $150 \mathrm{~mm}$.

\section{Motor speed controller}

The circuit used in this research uses the PID method to control the speed of the motors. This circuit can be divided into four main sections with specific tasks: The first section generates the sinusoidal wave; the second section is the controller; the third section is the motor starter; and the fourth section is the circuit feedback. To generate the desired waveform, an ATmega16A microcontroller is utilized. This microcontroller is able to generate PWM waves using built-in timers which contain the defined waveform. Then, this signal is passed through a low-pass filter such that the output is the desired waveform. The communication with the microcontroller and its programming are performed using a computer and a programmer. In this study, the AVRISP mkII communication protocol is connected to the computer using a USB cable. The programming language for the microcontroller is $\mathrm{C}$ and it is written using the CodeVisionAVR software. Programming

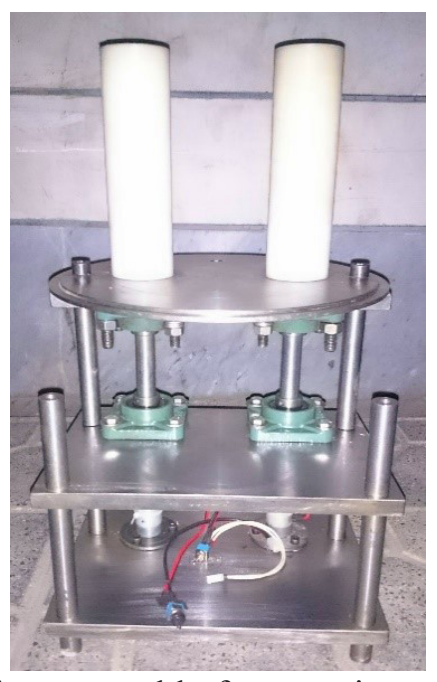

Figure 2. Mixer assembly for experimental tests.

is performed with the help of AVR studio. Figure 2 shows the assembly model of the chaotic mixer.

\section{Supplying the studied dye and fluid}

Glycerin $99.7 \%$ (Merc) with the density of 1200 $\mathrm{kg} / \mathrm{m}^{3}$ and viscosity of $0.81 \mathrm{~Pa} . \mathrm{s}$ is used as the studied fluid. To study the performance of the mixer, fluorescent powder (Fluorescent powder - Ukseung Chemical Co., Ltd) was completely ground using a planetary mill, combined with water, and mixed for 2 hours using a mechanical mixer such that the fluorescent powder (with the ratio of $30 \mathrm{~g}$ of glycerin, 1 gram of water and 1 gram of fluorescent powder) was uniformly distributed in the water. The studied glycerin fluid was mixed with water with the proportion of 38 to 1 and the prepared dye was injected into the glycerin. The results of the experiment were satisfactory and the prepared dye was entrapped in the middle of the glycerin for $10 \mathrm{~min}$.

\section{Experimental test of mixing}

Using a syringe, $2 \mathrm{cc}$ of dye was injected in the middle depth, $10 \mathrm{~cm}$ away from the center of the mixer, and pictures were taken every $4 \mathrm{~s}$. The picture of the samples was recorded using a Canon PowerShot G10 camera with 14.7 mega-pixels' resolution. Figure 3 shows the preparation and lighting of the chaotic mixer to perform the experimental tests. UV lamps (Noor Lamp Company) were used to illuminate the prepared dyes with fluorescent powder during photography. Figure 4 shows the initial position of the dye injected inside the mixer.

\section{Experimental results}

Figure 5 presents the dye mixing variations with time for the three Modes of rotational speeds in the same direction in $600 \mathrm{~s}$. As observed in Figure 5, in Modes 1 and 2, the dye injected did not distribute in the mixer area. The fluid elements existing in this area 

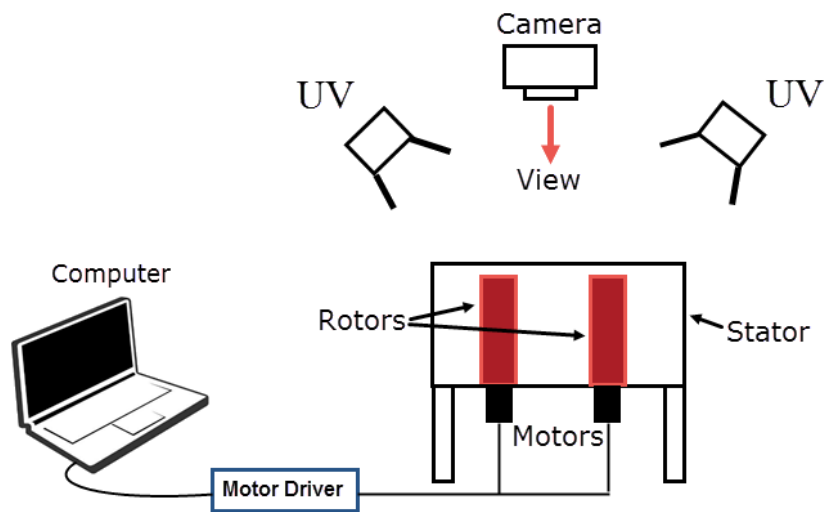

Figure 3. The schematic image of the chaotic mixer in the experimental test.

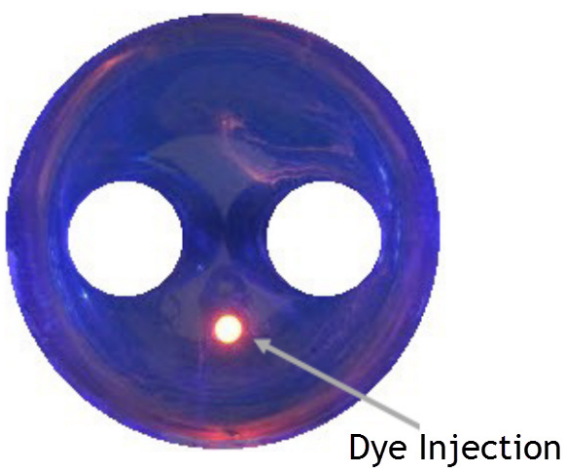

Figure 4. Dye injection position.

do not have any contact with other fluid elements and mixing is not performed well. The sinusoidal varying rotational speed is such that the rotor speed changes in the opposite direction, i.e., when the speed of one rotor increases, the speed of the other one decreases. The perturbations applied to the speed of the rotors lead to the generation of a chaotic advection and homogeneous mixing.

Figure 6 demonstrates the dye mixing in terms of time for the second three modes with the rotation of the rotor in the opposite direction for $600 \mathrm{~s}$. In the fourth mode where the rotors rotate with an equal rotational speed of $20 \mathrm{rpm}$, dye mixing is improved compared to that of the mode with rotations in the same direction. However, areas with a poor mixing are still observed. In the fifth mode, the rotational speeds in the left and right rotors with the values of $10 \mathrm{rpm}$ and $30 \mathrm{rpm}$, respectively, improve fluid particle mixing due to smaller dead zones. However, given the constant rotor speeds and steady flow, there is no possibility for the particles to move out of the secondary flows. In the sixth mode, the variations of the rotational speeds of the rotors in terms of time make the flow transient. This causes the small variations in this flow to grow, leading to chaotic flows. As observed in Figure 6, the dye is spread on all surfaces of the mixer in $600 \mathrm{~s}$, implying the elimination of the dead mixing zones and uniform advection of the fluid particles in the chaotic state.
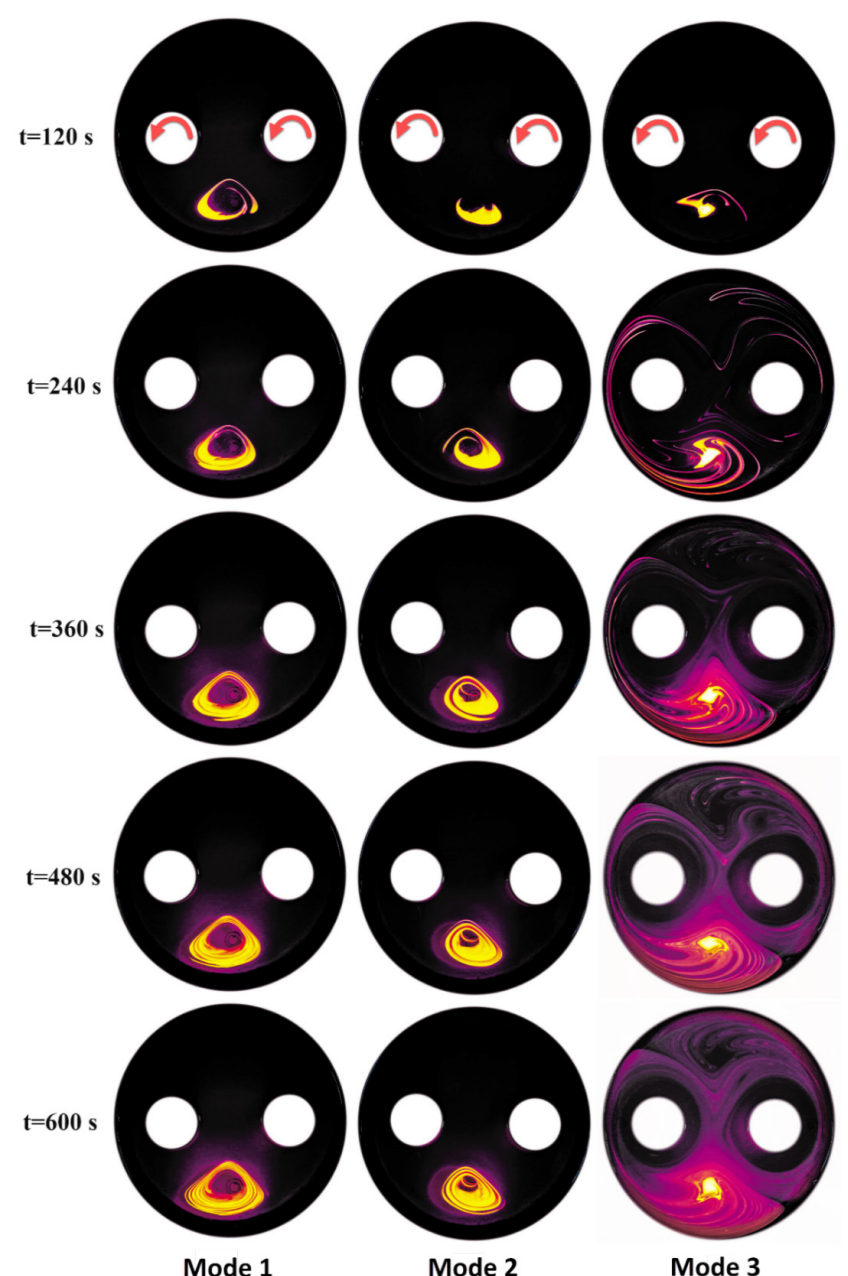

Mode 3

Figure 5. The mixing of the injected dye in terms of time for the modes in which rotors moved in the same direction. Mode $1\left(\mathrm{U}_{1}=20 \mathrm{rpm}, \mathrm{U}_{2}=20 \mathrm{rpm}, \varepsilon_{1}=0\right.$, $\left.\varepsilon_{2}=0\right)$, Mode $2\left(\mathrm{U}_{1}=10 \mathrm{rpm}, \mathrm{U}_{2}=30 \mathrm{rpm}, \varepsilon_{1}=0, \varepsilon_{2}=\right.$ $0)$, Mode $3\left(\mathrm{U}_{1}=20 \mathrm{rpm}, \mathrm{U}_{2}=20 \mathrm{rpm}, \varepsilon_{1}=0, \varepsilon_{2}=0.5\right)$.

\section{Mixing index}

Using the image processing method, it was attempted to quantify the mixing inside the mixer. To this end, a combination of a computer, a digital camera, and a processing code was used to measure the advection of the injected dye. The image generated this way was then taken into MATLAB software. In this software, the circular image was enclosed in a square frame. The images taken in the MATLAB environment are in the RGB space which should be converted to black and white images with a threshold to define a mixing index as the ratio of the number of white pixels to number of total pixels. In this mode, 0 indicates absolute black and 1 indicates absolute white. Figure 7 illustrates the processed image of six mixing modes at $t=180 \mathrm{~s}$. By determining the number of black and white pixels in each image, one can introduce a criterion of the mixing index that can quantify the mixing inside the mixer. Thus, the mixing index (MI) (Hosseinalipour et al., 2014) was used as a criterion for the quantification 


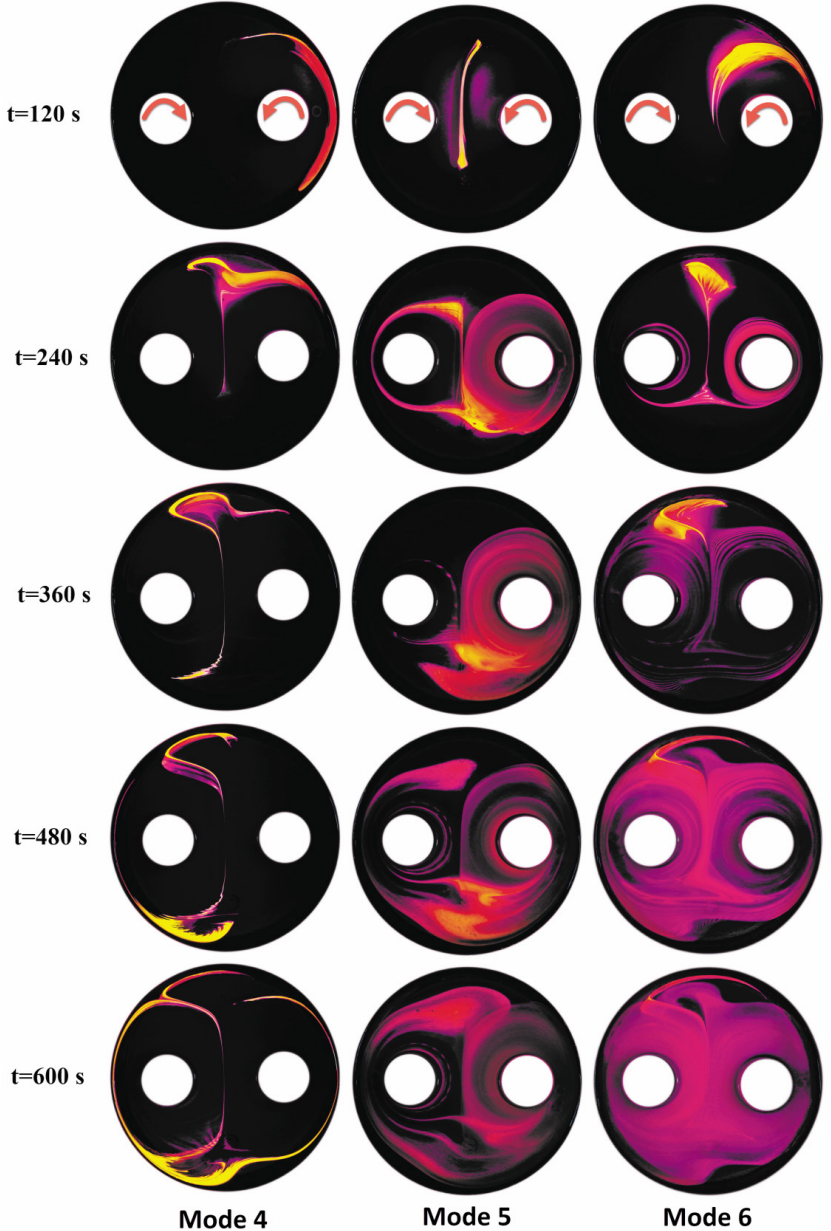

Figure 6. The mixing of the injected dye in terms of time for the modes in which the rotors rotate in opposite directions. Mode $4\left(\mathrm{U}_{1}=20 \mathrm{rpm}, \mathrm{U}_{2}=-20\right.$ $\left.\mathrm{rpm}, \varepsilon_{1}=0, \varepsilon_{2}=0\right)$, Mode $5\left(\mathrm{U}_{1}=10 \mathrm{rpm}, \mathrm{U}_{2}^{2}=-30\right.$ rpm, $\left.\varepsilon_{1}=0, \varepsilon_{2}=0\right)$, Mode $6\left(\mathrm{U}_{1}=20 \mathrm{rpm}, \mathrm{U}_{2}=-20\right.$ rpm, $\varepsilon_{1}=0, \varepsilon_{2}=0.5$ ).

of the mixing. Mixing index requires the total number of pixels and their size in comparison with the size of the mixer.

$\mathrm{MI}=\frac{\text { Number of white pixels }}{\text { Total number of pixels }}$

The variations of the mixing index in terms of time are depicted in Figure 8. It is clear that the mixing is not significantly different in the second

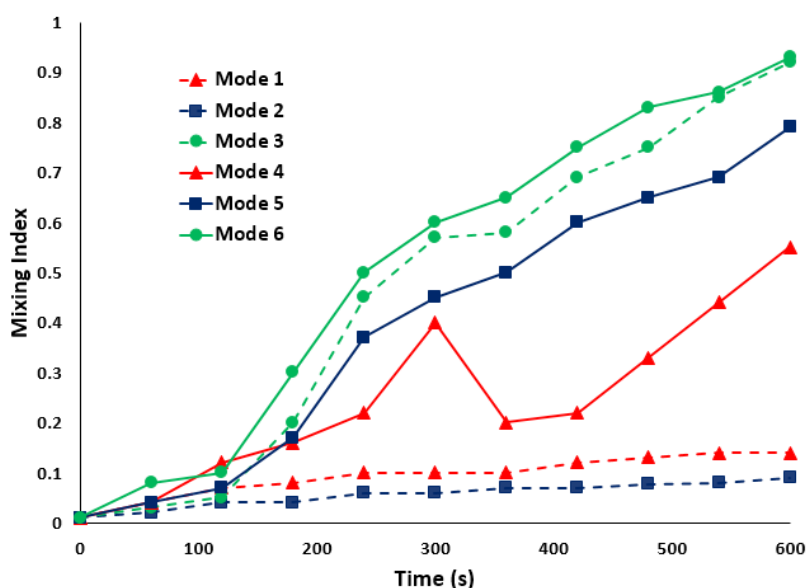

Figure 8. The variations of mixing index in terms of time for six mixing modes.

mode compared to the first mode where the rotors have unequal speeds. In the second mode, the rotating flow has become smaller compared to that of the first mode. Therefore, the area in which the dye is spread is smaller. As a result, the mixing index shows a smaller value for the second mode compared to the first mode. In the third mode with sinusoidal rotor speeds, the flow has become chaotic and mixing is considerably increased such that the mixing index in the third mode has increased almost 10 times compared to the first and second modes. Rotations in opposite directions in the fourth mode increased the mixing compared to the first mode with rotors rotating in the same direction. However, it is still less than the mixing in the third mode in which the chaotic flow is due to the sinusoidal but unidirectional rotation of rotors. The mixing in the fifth mode in which the rotors rotate with unequal speeds in opposite directions is the highest compared to the other modes in which the rotors have a constant speed. The mixing index in the fifth mode is almost the same as that of the third mode with a chaotic flow. This shows that mixing can be considerably increased with constant rotor speed. However, to maximize the mixing, the steady flow is not efficient enough since the flow becomes chaotic in the sixth mode where rotors rotate with sinusoidal speeds and in opposite directions, and this causes the mixing to reach its maximum value in the same duration compared to the other modes. One can conclude that, with similar

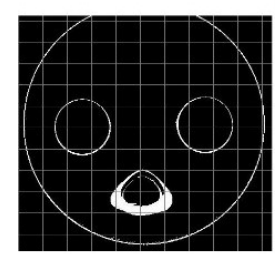

Mode 1

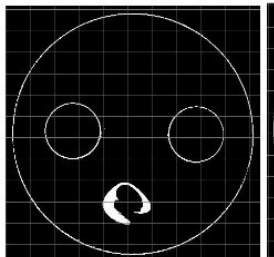

Mode 2

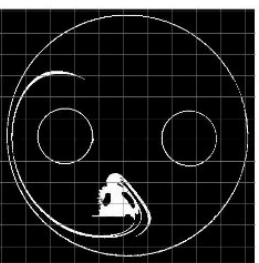

Mode 3

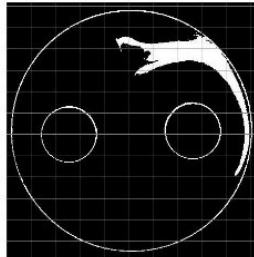

Mode 4

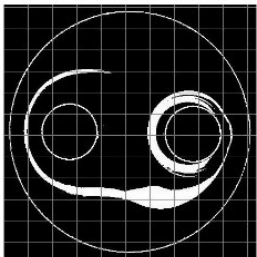

Mode 5

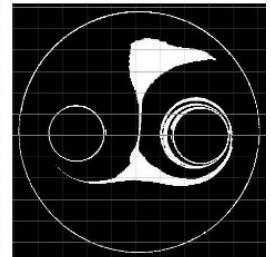

Mode6

Figure 7. The processed images in six mixing modes at $\mathrm{t}=180 \mathrm{~s}$. 
boundary conditions, chaotic flows are more efficient compared to non-chaotic modes since one of the most important mixing parameters in highly viscous fluids is the time it takes for the fluid to be mixed homogeneously. In this regard, the chaotic flow is deemed a proper approach for resolving the issue of mixing in highly viscous flows.

\section{NUMERICAL SOLUTION}

\section{Numerical method}

Due to the low Reynolds number and the small value of the ratio of rotor and stator radius to the batch mixer depth, the mixing in the third dimension is neglected and the simulation is performed in two dimensions. The main governing equations of the viscous flow are as follows:

$\frac{\partial \rho}{\partial t}+\operatorname{div}(\rho V)=0$

$\rho \frac{\mathrm{DV}}{\mathrm{Dt}}=-\nabla \mathrm{P}+\mu \nabla^{2} \mathrm{~V}$

where $\rho$ denotes the density of the fluid, $\mathrm{V}$ indicates the velocity of the fluid, $P$ represents the pressure, and $\mu$ shows the viscosity of the fluid.

The non-dimensional form of equation (9) is:

$\mathrm{St} \frac{\mathrm{DV}^{*}}{\mathrm{Dt}^{*}}+\left(\mathrm{V}^{*} . \nabla\right) \mathrm{V}^{*}=-\mathrm{Eu} \mathrm{P}^{*}+\frac{1}{\mathrm{Re}} \nabla^{2} \mathrm{~V}^{*}$

where $\mathrm{Eu}$ is the Euler number, Re is the Reynolds number and St is the Strouhal number. Since the Strouhal number in Equation (4) is in the range of 0.09 to 0.012 , the problem can be solved as a quasi-steady flow problem.

Equations (8-9) are discretized using the SUPG method with the assumption of laminar flow and the momentum equation system is discretized using the TDMA method, while the pressure equations are calculated using PCG. The Cartesian coordinate system is used for the simulation based on the finite-element method. The convergence criterion is calculated as follows for each degree of freedom at each iteration:

$\mathrm{M}_{\varnothing}=\frac{\sum_{\mathrm{i}=1}^{\mathrm{N}}\left|\varnothing_{\mathrm{i}}^{\mathrm{k}}-\varnothing_{\mathrm{i}}^{\mathrm{k}-1}\right|}{\sum_{\mathrm{i}=1}^{\mathrm{N}}\left|\varnothing_{\mathrm{i}}^{\mathrm{k}}\right|} \leq 10^{-8}$

where $\varnothing$ is the degree of freedom for the pressure and velocity of the flow, $\mathrm{N}$ is the total number of finiteelement nodes, and $\mathrm{k}$ is the number of the current iteration. The convergence criterion $\mathrm{M}_{\varnothing}$ indicates the variations of the variable from the current $\mathrm{k}^{\text {th }}$ and the previous $(\mathrm{k}-1)^{\text {th }}$ iterations which is normalized by dividing it by its current value. The convergence criterion is applied to all $\mathrm{N}$ finite-element nodes. The set of governing equations was solved using ANSYS (FLOTRAN) software.

\section{Grid independence check}

Four meshes $(833,1088,1377$, and 1700 elements) were used to solve the flow field and investigate the grid independence test. The results of comparison of velocities at the 8 nodes numbered in Figure (9) are presented in Figure (10). Simulations were carried out with 1377 elements since the difference in speed between the meshes of 1377 and 1700 elements had an error of less than $1 \%$.

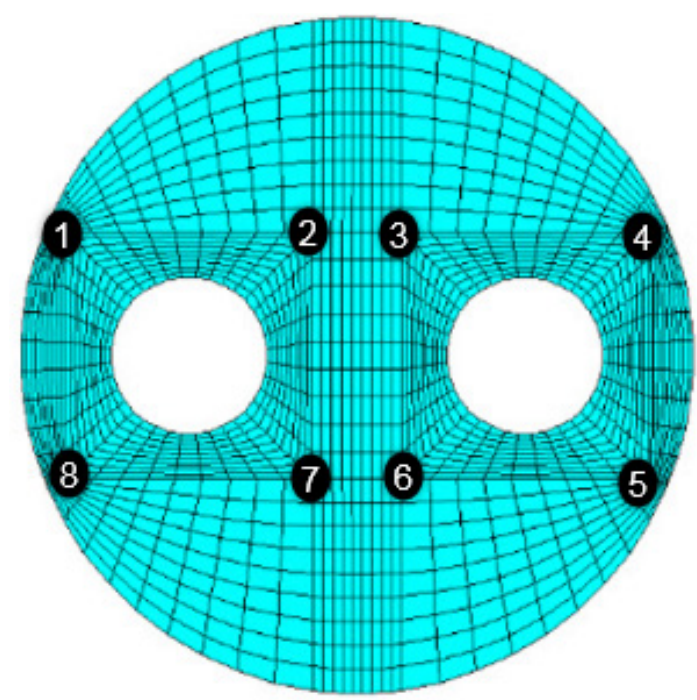

Figure 9. Mesh of mixer geometry.

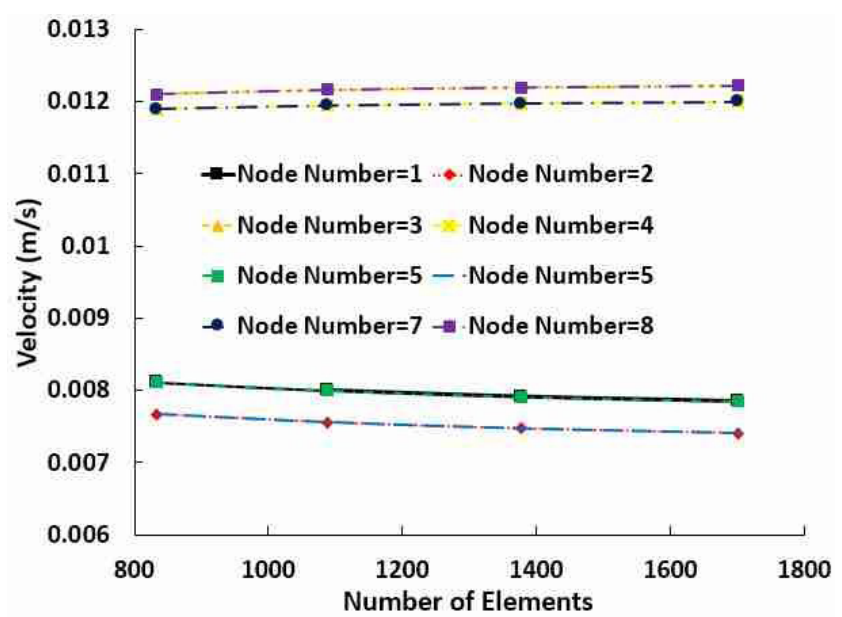

Figure 10. Mesh independence study.

\section{Particle tracing and validation}

The fourth-order Runge-Kutta method was utilized for integrating the system of advection equations (12) in order to perform the Lagrangian particle tracing, and the particle position was determined over time after the Eulerian solution of the flow. 
To validate the numerical results, the flow inside the mixer was simulated for different conditions. Then, 400 fluid particles with the initial arrangement of the circle and the same position as the injected color were traced in the experimental test as Lagrangian. Figure 11 shows the comparison of numerical and experimental results at the same time for modes 5-6. As can be seen, the results were in very good agreement, which indicated the high accuracy of the numerical calculations.

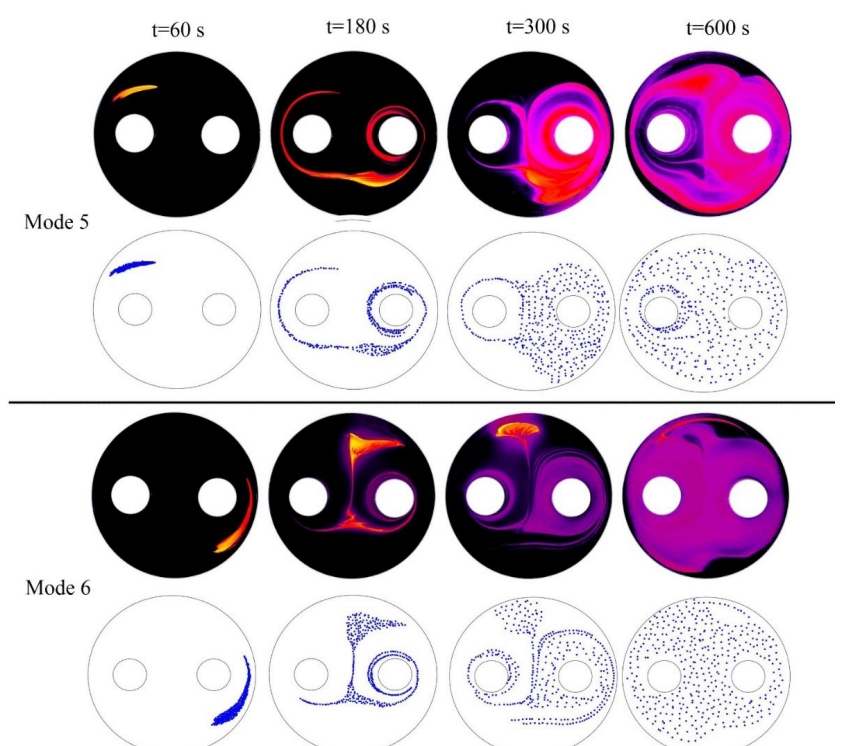

Figure 11. Comparing the experimental and numerical results of mixing for Mode $5\left(\mathrm{U}_{1}=10 \mathrm{rpm}, \mathrm{U}_{2}=-30\right.$ rpm, $\left.\varepsilon_{1}=0, \varepsilon_{2}=0\right)$, Mode $6\left(\mathrm{U}_{1}=20 \mathrm{rpm}, \mathrm{U}_{2}^{2}=-20\right.$ rpm, $\varepsilon_{1}=0, \varepsilon_{2}=0.5$ ).

\section{Numerical results}

Figure 12 illustrates the streamlines in Mode 1. In this mode, there are one hyperbolic point and four elliptic points. The elliptic points are the points of the flow around which the flow rotates as a fixed point and does not leave. The fact that fluid particles do not leave these areas leads to the modifications of chaotic flows and the formation of islands that the fluid particles do not enter or leave and are considered as dead mixing zones.

In order to improve mixing, a geometrical or velocity perturbation should be applied in the boundary conditions to displace the elliptic and hyperbolic points. In Mode 2, rotor speeds become unequal but still in the same direction. In Modes 4 and 5 , rotor speeds are similar to those of Modes 1 and 2 but with opposing rotating directions. Figure 13 shows the streamlines inside the mixer for these modes. Although the different rotating directions of rotors lead to increased mixing, the location of the flow fixed points such as hyperbolic and saddle points does not change with time, given that the flow inside the mixer is steady. The flow's independence of time leads to a

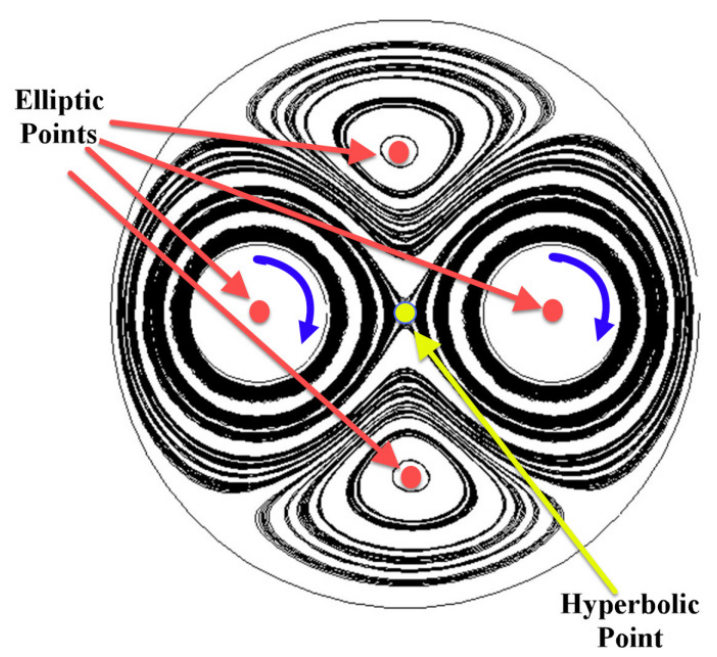

Figure 12. Streamlines in Mode $1\left(\mathrm{U}_{1}=20 \mathrm{rpm}, \mathrm{U}_{2}=\right.$ $20 \mathrm{rpm}, \varepsilon_{1}=0, \varepsilon_{2}=0$ ).
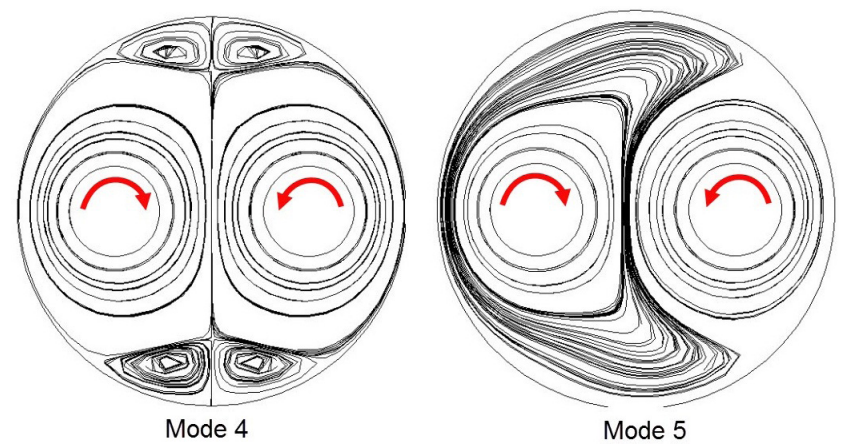

Figure 13. The numerical results for the streamlines of equal speeds and unequal rotor speeds. Mode $4\left(\mathrm{U}_{1}=\right.$ $\left.20 \mathrm{rpm}, \mathrm{U}_{2}=-20 \mathrm{rpm}, \varepsilon_{1}=0, \varepsilon_{2}=0\right)$, Mode $5\left(\mathrm{U}_{1}=10\right.$ rpm, $U_{2}=-30 \mathrm{rpm}, \varepsilon_{1}=0, \varepsilon_{2}=0$ ).

non-chaotic flow since, in 2D flows, the flow should be a function of time in order to create chaotic particle movements as the $2 \mathrm{D}$ steady advection is integrable. In these modes, the fluid particles move in periodic paths and optimal mixing does not happen.

Figure 14 illustrates the streamlines inside the mixer for Modes 3 and 6 (sinusoidal varying rotational speed). Upon applying a perturbation in the streamline by making the rotational speeds of the rotors sinusoidal, the location of the fixed points changes with time and inclines to the left or right which, in turn, causes the particles to move away from the secondary flow. The hyperbolic point is displaced due to oscillating speeds of the two rotors. The existence of hyperbolic points and the behavior of their manifolds is one of the conditions for the beginning of mixing because, when the location of the fixed point is changed, the previous stable or unstable manifolds intersect the current unstable or stable manifolds and this intersection leads to frequent stretching and folding.

To review the mixing quality and sensitivity of the initial flow conditions, a Poincare section can be 


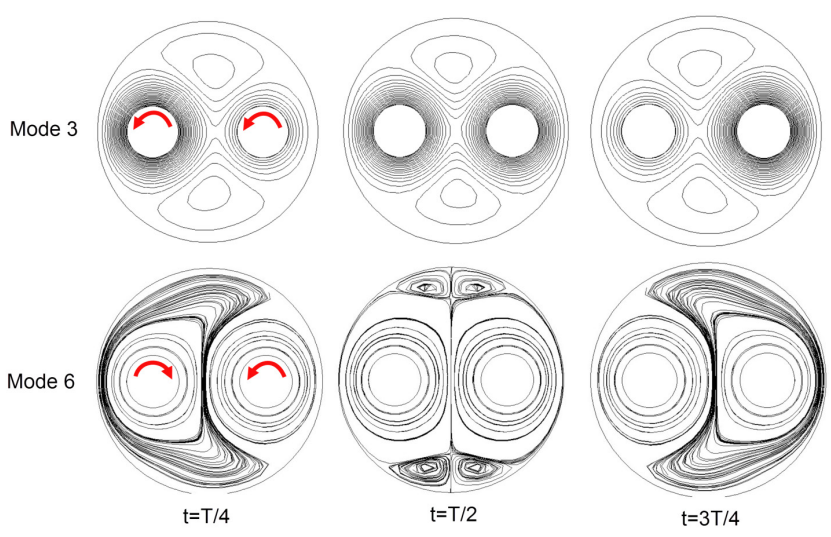

Figure 14. The numerical results for the sinusoidal varying rotational speed for Mode $3\left(\mathrm{U}_{1}=20 \mathrm{rpm}, \mathrm{U}_{2}\right.$ $\left.=20 \mathrm{rpm}, \varepsilon_{1}=0, \varepsilon_{2}=0.5\right)$ and Mode $6\left(\mathrm{U}_{1}=20 \mathrm{rpm}\right.$, $\mathrm{U}_{2}=-20 \mathrm{rpm}, \varepsilon_{1}=0, \varepsilon_{2}=0.5$ ).

used. To create a Poincare section, 400 particles of fluid with a circular arrangement in the coordinates of the color injected into the experimental tests for the period of $600 \mathrm{sec}$ were tracked and the position of the particle was recorded every $3 \mathrm{sec}$. Figure 15 shows the position of 400 particles over $600 \mathrm{sec}$ for all the modes. As can be seen, in modes 1-2 and 3-4, in which the rotors had constant rotational velocity and the flow in the mixer was non-chaotic, the particles were trapped in vortex and island flows and only spread in a small part of the mixer. The poor distribution in the
Poincare sections indicated the poor mixing of a nonchaotic mixer.

The sinusoidal velocity perturbation in terms of time in the chaotic mixer enabled the fluid particles to be released from dead-flow traps. The path of the particles was exponentially distanced and, therefore, there was more uniform distribution than in the typical mixer.

\section{CONCLUSION}

In this study, the effects of unequal speeds and rotations in opposite directions were numerically and experimentally studied on the mixing of highly viscous fluids and compared with chaotic flow. Six modes were tested for this purpose: In the first mode, the rotors rotated in the same direction with constant and equal speeds; in the second mode, the rotors rotated in the same direction with constant but different speeds; in the third mode, the rotors rotated in the same direction but in the form of a sinusoidal function of time; the other three modes, i.e. Modes 4, 5, and 6, were the same as the first three modes but with rotors rotating in opposite directions.

Results of numerical simulations showed that, in Modes 1, 2, 4, and 5 where the flow is not timedependent, fixed points exist in the flow which lead to secondary flows and less mixing. In order to

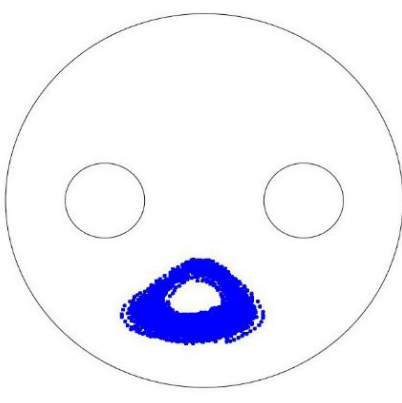

Mode 1

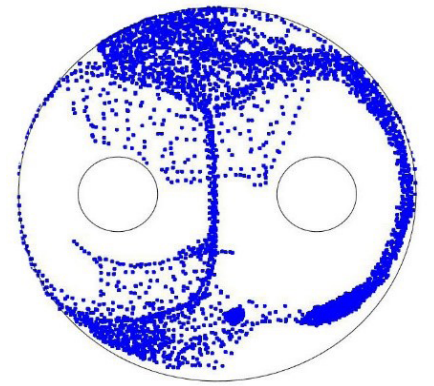

Mode 4

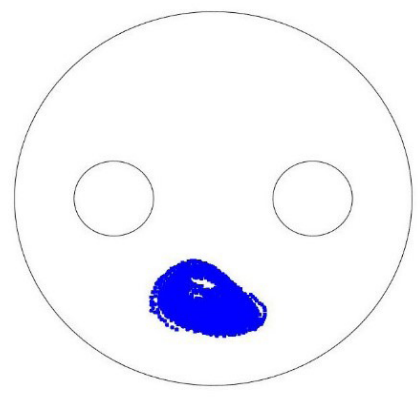

Mode 2

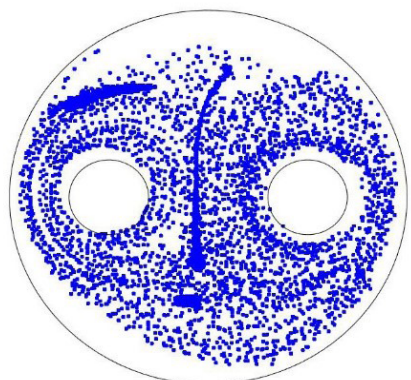

Mode 5

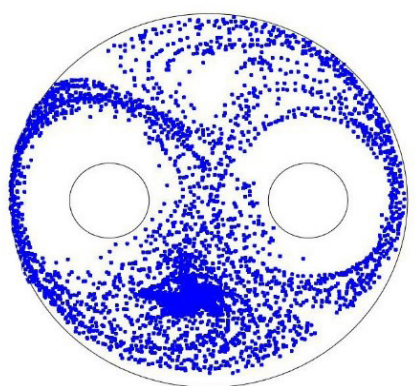

Mode 3

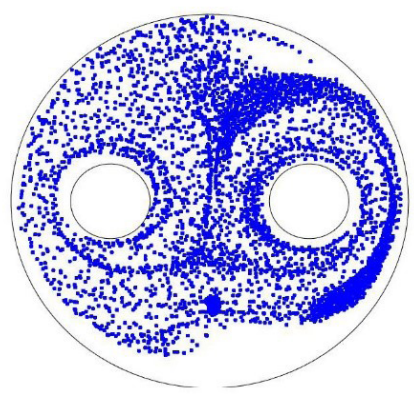

Mode 6

Figure 15. Poincare section of fluid particle trajectories for Mode $1\left(U_{1}=20 \mathrm{rpm}, \mathrm{U}_{2}=20 \mathrm{rpm}, \varepsilon_{1}=0, \varepsilon_{2}=0\right)$, Mode $2\left(\mathrm{U}_{1}=10 \mathrm{rpm}, \mathrm{U}_{2}=30 \mathrm{rpm}, \varepsilon_{1}=0, \varepsilon_{2}=0\right)$, Mode $3\left(\mathrm{U}_{1}=20 \mathrm{rpm}, \mathrm{U}_{2}=20 \mathrm{rpm}, \varepsilon_{1}=0, \varepsilon_{2}=0.5\right)$, Mode $4\left(\mathrm{U}_{1}=20\right.$ $\left.\mathrm{rpm}, \mathrm{U}_{2}=-20 \mathrm{rpm}, \varepsilon_{1}=0, \varepsilon_{2}=0\right)$, Mode $5\left(\mathrm{U}_{1}=10 \mathrm{rpm}, \mathrm{U}_{2}=-30 \mathrm{rpm}, \varepsilon_{1}=0, \varepsilon_{2}=0\right)$ and Mode $6\left(\mathrm{U}_{1}=20 \mathrm{rpm}\right.$, $\left.\mathrm{U}_{2}=-20 \mathrm{rpm}, \varepsilon_{1}=0, \varepsilon_{2}=0.5\right)$. 
experimentally study the effect of unequal speeds and different rotating directions of the rotor, dye was injected into the glycerin fluid and the mixing was examined by taking pictures of the mixing of the fluid. The experimental results revealed that, in modes where the rotors rotate in the same direction, unequal rotor speeds do not significantly affect the mixing. However, when the direction of the rotors is opposite, mixing is improved, particularly when the rotors have unequal speeds in the opposite direction. On the other hand, the location of the fixed point does not change and the particles inside the generated secondary flows move in a periodic path, given that the flow is not transient. Nevertheless, varying the rotors' speed leads to the generation of a chaotic flow, which makes the mixing more efficient compared to the other nonchaotic modes where the rotors either rotate in the same or in opposite directions.

\section{REFERENCES}

Aguirre, A., Castillo, E., Cruchaga, M., Codina, R., Baiges, J. Stationary and time-dependent numerical approximation of the lid-driven cavity problem for power-law fluid flows at high Reynolds numbers using a stabilized finite element formulation of the VMS type. Journal of Non-Newtonian Fluid Mechanics, 257, 22-43 (2018). https://doi. org/10.1016/j.jnnfm.2018.03.014

Aref, H. J. P. O. F. The development of chaotic advection. 14, 1315-1325 (2002). https://doi. org/10.1063/1.1458932

Ballal, B., Rivlin, R. Flow of a Newtonian fluid between eccentric rotating cylinders: Inertial effects. Archive for Rational Mechanics and Analysis, 62, 237-294 (1976). https://doi.org/10.1007/BF00280016

El Omari, K., Le Guer, Y. Alternate rotating walls for thermal chaotic mixing. International Journal of Heat and Mass Transfer, 53, 123-134 (2010). https:// doi.org/10.1016/j.ijheatmasstransfer.2009.09.046

Galaktionov, O., Meleshko, V., Peters, G., Meijer, H. Stokes flow in a rectangular cavity with a cylinder. Fluid Dynamics Research, 24, 81-102 (1999). https://doi.org/10.1016/S0169-5983(98)00013-6

Grosso, G., Hulsen, M. A., Sarhangi Fard, A., Overend, A., Anderson, P. D. Mixing processes in the cavity transfer mixer: A thorough study. AIChE Journal, 64, 1034-1048 (2018). https://doi.org/10.1002/ aic. 15986

Hosseinalipour, S. M., Tohidi, A., Mashaei, P. R., Mujumdar, A. S. Experimental investigation of mixing in a novel continuous chaotic mixer. Korean Journal of Chemical Engineering, 31, 1757-1765 (2014). https://doi.org/10.1007/s11814014-0071-x
Hosseinalipour, S. M., Tohidi, A., Shokrpour, M., Nouri, N. M. Introduction of a chaotic dough mixer, part A: mathematical modeling and numerical simulation. Journal of Mechanical Science and Technology, 27, 1329-1339 (2013). https://doi. org/10.1007/s12206-012-0895-4

Jana, S. C., Metcalfe, G., Ottino, J. Experimental and computational studies of mixing in complex Stokes flows: the vortex mixing flow and multicellular cavity flows. Journal of Fluid Mechanics, 269, 199-246 (1994). https://doi.org/10.1017/ S0022112094001539

Jeffery, G. B. The rotation of two circular cylinders in a viscous fluid. Proc. R. Soc. Lond. A, 101, 169-174 (1922). https://doi.org/10.1098/rspa.1922.0035

Jegatheeswaran, S., Ein-Mozaffari, F., Wu, J. Process intensification in a chaotic SMX static mixer to achieve an energy-efficient mixing operation of non-newtonian fluids. Chemical Engineering and Processing: Process Intensification, 124, 1-10 (2018). https://doi.org/10.1016/j.cep.2017.11.018

Jung, S. Y., Ahn, K. H., Kang, T. G., Park, G. T., Kim, S. U. Chaotic mixing in a barrier-embedded partitioned pipe mixer. AIChE Journal, 64, 717-729 (2018). https://doi.org/10.1002/aic.15929

Lefevre, A., Mota, J., Rodrigo, A., Saatdjian, E. Chaotic advection and heat transfer enhancement in Stokes flows. International Journal of Heat and Fluid Flow, 24, 310-321 (2003). https://doi. org/10.1016/S0142-727X(03)00022-5

Leprevost, J.-C., Lefévre, A., Brancher, J.-P., Saatdjian, E. Chaotic mixing and heat transfer in a periodic 2D flow. Comptes Rendus de l'Academie des Sciences Series IIB Mechanics Physics Chemistry Astronomy, 9, 519-526 (1997). https:// doi.org/10.1016/S1251-8069(97)89076-8

Luan, D., Chen, Y., Wang, H., Wang, Y., Wei, X. Chaotic characteristics of pseudoplastic fluid induced by 6PBT impeller in a stirred vessel. Chinese Journal of Chemical Engineering, 27, 293-297 (2018). https://doi.org/10.1016/j.cjche.2018.06.001

Mizuno, Y., Funakoshi, M. Chaotic mixing due to a spatially periodic three-dimensional flow. Fluid Dynamics Research, 31, 129-149 (2002). https:// doi.org/10.1016/S0169-5983(02)00093-X

Mizuno, Y., Funakoshi, M. Chaotic mixing caused by an axially periodic steady flow in a partitionedpipe mixer. Fluid Dynamics Research, 35, 205-227 (2004). https://doi.org/10.1016/j. fluiddyn.2004.05.003

Msaad, A. A., Mahdaoui, M., Kousksou, T., Allouhi, A., El Rhafiki, T., Jamil, A., Ouazzani, K. Numerical simulation of thermal chaotic mixing in multiple rods rotating mixer. Case Studies in Thermal Engineering, 10, 388-398 (2017). https:// doi.org/10.1016/j.csite.2017.09.005 
Niederkorn, T. C., Ottino, J. M. Chaotic mixing of shearthinning fluids. AIChE Journal, 40, 1782-1793 (1994). https://doi.org/10.1002/aic.690401103

Ottino, J. M., Ottino, J. The kinematics of mixing: stretching, chaos, and transport, Cambridge University Press (1989).

Pacheco, J. R., Chen, K. P., Hayes, M. A. Rapid and efficient mixing in a slip-driven three-dimensional flow in a rectangular channel. Fluid Dynamics Research, 38, 503-521 (2006). https://doi. org/10.1016/j.fluiddyn.2006.03.003

Shirmohammadi, F., Tohidi, A. Mixing enhancement using chaos theory in fluid dynamics: Experimental and numerical study. Chemical Engineering Research and Design, 141, 350-360 (2018). https:// doi.org/10.1016/j.cherd.2018.04.007

Swanson, P., Ottino, J. A comparative computational and experimental study of chaotic mixing of viscous fluids. Journal of Fluid Mechanics, 213, 227-249 (1990). https://doi.org/10.1017/ S0022112090002300

Tohidi, A., Hosseinalipour, S., Monfared, Z. G., Mujumdar, A. Laminar Heat Transfer Enhancement Utilizing Nanofluids in a Chaotic Flow. Journal of Heat Transfer, 136, 1-8 (2014). https://doi. org/10.1115/1.4027773
Tohidi, A., Hosseinalipour, S., Shokrpour, M., Mujumdar, A. Heat transfer enhancement utilizing chaotic advection in coiled tube heat exchangers. Applied Thermal Engineering, 76, 185-195 (2015). https://doi.org/10.1016/j. applthermaleng.2014.10.073

Tohidi, A., Hosseinalipour, S., Taheri, P., Nouri, N., Mujumdar, A. Chaotic advection induced heat transfer enhancement in a chevron-type plate heat exchanger. Heat and Mass Transfer, 49, 1535-1548 (2013). https://doi.org/10.1007/s00231-013-11805

Wünsch, O., Böhme, G. Numerical simulation of $3 \mathrm{~d}$ viscous fluid flow and convective mixing in a static mixer. Archive of Applied Mechanics, 70, 91-102 (2000). https://doi.org/10.1007/s004199900042

Xu, B.-P., He, L., Wang, M.-G., Tan, S.-Z., Yu, H.-W., Turng, L.-S. Numerical Simulation of Chaotic Mixing in Single Screw Extruders with Different Baffle Heights. International Polymer Processing, 31, 108-118 (2016). https://doi. org/10.3139/217.3167

Zaslavsky, G. Lagrangian turbulence and anomalous transport. Fluid Dynamics Research, 8, 127133 (1991). https://doi.org/10.1016/01695983(91)90036-I 
